\title{
La influencia de la ira y la depresión como posibles factores de riesgo en la adaptación a la enfermedad cardiovascular
}

\author{
Esperanza Valls Lázaro y Beatriz Rueda Laffond \\ Hospital Nuestra Señora de Gracia, Zaragoza, Universidad Nacional de Educación a Distancia \\ Correspondencia: Esperanza Valls Lázaro. Hospital Nuestra Señora de Gracia. \\ C./ Panamá, 4 - 50012 Zaragoza. Correo electrónico: evalls@salud.aragon.es
}

\section{RESUMEN}

El presente estudio tuvo como objetivos: (I) examinar, en una muestra de pacientes cardiovasculares, las diferencias en función del sexo en relación con: la ira y depresión; las estrategias de afrontamiento; la calidad de vida (CV); y con dos criterios cardiovasculares (enfermedad coronaria y factores de riesgo), (II) estudiar la capacidad predictiva de la ira y la depresión con respecto a los criterios cardiovasculares, las estrategias de afrontamiento y la CV. Los participantes fueron 107 pacientes (edad media $=63$ años; $60 \%$ hombres y $40 \%$ mujeres). Los resultados muestran que los hombres presentaron mayor frecuencia de infarto y de factores de riesgo; mientras que las mujeres indicaron otros trastornos cardiovasculares, y un estado de ánimo más negativo. La depresión predijo mayor probabilidad de padecer alguna enfermedad coronaria, y una CV más baja. Tanto la ira como la depresión se asociaron con estrategias de afrontamiento más emocionales, aunque el peso de la depresión fue superior.

Palabras clave: Enfermedad cardiovascular, factor de riesgo, depresión, ira, afrontamiento, calidad de vida.

\section{ABSTRACT}

This study had the following aims: (I) to examine, in a sample of cardiovascular patients, gender differences in: anger and depression, coping strategies, quality of life (QoL); and two cardiovascular criteria (coronary disease and risk factors); and (II) to study the predictive capacity of anger and depression in relation to both cardiovascular criteria, coping strategies and QoL. Participants were one hundred and seven patients (Mean age $=63$ years; $60 \%$ males and $40 \%$ females). The results show that the men had a higher rate of myocardial infarction and risk factors; whereas females indicated to suffer from other cardiovascular conditions and to have more negative mood state. Depression predicted a higher risk of coronary disease and lower QoL. Anger and depression were both associated with emotion focused coping, although the weight of depression was higher than the one of anger.

Keywords: Cardiovascular disease, risk factors, depression, anger, coping, quality of life. 
Introducción

El padecimiento de una enfermedad cardiovascular (ECV) es una de las principales causas de muerte y de incapacitación en muchos países del mundo. De hecho, según la Organización Mundial de la Salud (2008) las ECV son la causa de más de 17,5 millones de muertes al año.

Diferentes estudios (Everson-Rose y Lewis, 2005; Fernández-Abascal et al., 2003; Kubzansky y Kawachi, 2000; Ruiz et al., 2008; Wielgosz y Nolan, 2000) han destacado la importancia de identificar los determinantes que influyen sobre el malestar psicológico de los pacientes con ECV. La definición de estos aspectos podría contribuir a amortiguar dicho malestar y a mejorar la calidad de vida, y el pronóstico de la enfermedad en estos pacientes.

Algunos de los factores que están siendo objeto de una mayor atención por parte de los investigadores, son los de tipo genético, como el sexo; los biológicos, como la hipertensión arterial y el colesterol; o los comportamentales, como los hábitos nocivos de salud.

Junto a estos elementos, otros de índole psicoemocional, tales como la depresión, la ira o la forma de afrontar el estrés, pueden tener un impacto negativo sobre la calidad de vida y el ajuste ante la ECV (Jacka et al., 2007; Lane et al., 2001; Stafford et al., 2007).

\section{Diferencias de sexo y enfermedad cardiovascular}

Si se atiende a las diferencias de sexo en el marco de la ECV, se ha constatado que las mujeres diagnosticadas con alguna patología cardiovascular suelen cuidar menos su salud, presentando una mayor comorbilidad e inadaptación, y una calidad de vida más baja (Emery et al., 2004). A pesar de su enfermedad, las mujeres tienden a seguir atendiendo las necesidades y demandas del ambiente familiar y de la casa, retornan menos a su trabajo habitual y desarrollan, en mayor medida, un estado de ánimo ansioso-depresivo. Asimismo, se ha apreciado que las estrategias de afrontamiento que ponen en marcha las mujeres con ECV, son más de tipo evitativo y emocional (Kristofferzon et al., 2003, 2005).

Si bien es cierto que el inicio de la ECV en el caso de las mujeres ocurre en una edad más avanzada, la probabilidad de reinfarto es también más alta, siendo el riesgo de muerte por esta causa dos veces mayor (Boutin-Foster y Charlson, 2007; Rueda y Pérez, 2006). La confluencia de estos aspectos se relaciona, además, con el hecho de que las mujeres cumplen, hasta en un 20\% menos, con la rehabilitación necesaria para lograr una mejoría en su ECV (Grace et al., 2002).

En su conjunto, estos estudios ponen de manifiesto el dato de que las diferencias de sexo, con respecto al ajuste a la ECV, tiene unas implicaciones que merecen ser consideradas con mayor detenimiento. Desde el punto de vista aplicado, es posible que la consideración de las diferencias de sexo pueda facilitar el diseño de intervenciones que estimulen a las mujeres a buscar antes, y a comprometerse en mayor medida, con su tratamiento médico.

Por ese motivo, uno de los objetivos planteados en nuestro estudio ha sido el de conocer en qué medida, en una muestra de pacientes cardiovasculares, podían diferir los hom- 
bres y las mujeres en relación con una serie de factores de riesgo cardiovascular y de aspectos psicoemocionales.

Estado de ánimo depresivo en los trastornos cardiovasculares

La depresión es uno de los principales factores de riesgo en el inicio y desarrollo de la ECV (Wulsin y Singal, 2003), asociado también a un incremento en la mortalidad en pacientes con un infarto de miocardio (IM) previo (Jacka et al., 2007). Se estima que un $20 \%$ de los individuos con algún tipo de trastorno cardiaco cumple criterios, según DSM-IV, de depresión mayor (Davidson y cols., 2006).

La depresión parece afectar al estado de salud de los pacientes con ECV a través de distintos mecanismos. Uno de estos mecanismos son los factores de riesgo. Diferentes investigaciones han evidenciado que la depresión se relaciona con un mayor número de factores de riesgo de tipo cardiovascular, como la hipercolesterolemia, la hipertensión, la diabetes, la obesidad y una mayor distribución central de la grasa en el cuerpo (Fenton y Stover, 2006; Frasure-Smith et al., 1995; Jacka et al., 2007; Lett et al., 2004).

Un segundo mecanismo sobre el que la depresión podría estar actuando en los pacientes con ECV, son las estrategias de afrontamiento. Así, por ejemplo, el empleo de estrategias de afrontamiento centradas en el problema, tales como el afrontamiento activo, la planificación, o el uso de apoyo instrumental, representan respuestas asociadas con mejores resultados a largo plazo (Doering et al., 2004; Holahan et al., 1997; Murberg et al., 2004). En oposición, la utilización del afrontamiento centrado en la emoción y la evitación, se ha relacionado con indicadores de malestar emocional, como la ansiedad, la depresión, la ira, y con sentimientos de incapacidad e inhibición social (Cameron, 2009; Murberg et al., 2004). Otros autores (Giltay et al., 2006), han señalado también que el tipo de afrontamiento que emplean los pacientes para hacer frente al estrés que genera la ECV, puede disminuir la sintomatología depresiva, tal sería el caso de la reevaluación positiva.

Finalmente, la calidad de vida representa, en el enfermo cardiovascular, otro ámbito que puede verse especialmente afectado por el estado emocional del paciente. Estudios recientes muestran que las emociones negativas, como la depresión y la ansiedad, predicen una calidad de vida más baja en personas que han sufrido IM, y en pacientes que se han sometido a la cirugía por bypass (Chung et al., 2009; Mayou et al., 2000; Stafford et al., 2007). Rueda y Pérez, (2006) encontraron que el apoyo social, definido como un componente de la calidad de vida, promovía una adherencia más alta al tratamiento médico, y un mayor número de cambios conductuales dirigidos a fomentar el bienestar físico y psicológico en pacientes con ECV.

De otro lado, parece haber un cierto acuerdo en que, conforme aumenta la calidad de vida del paciente, mayor es la probabilidad de supervivencia en los trastornos cardiacos crónicos (Birket-Smith et al., 2009; Kao, 2007). Algunos trabajos (Faller et al., 2007) han sugerido que la calidad de vida podría disminuir el estado de ánimo depresivo de los pacientes, y 
potenciar sus recursos sociales reduciendo así su aislamiento (Blom y cols., 2007; Rose y cols., 2005).

Ira-hostilidad y enfermedad cardiovascular

Junto a la depresión, la hostilidad representa otro factor psicosocial relacionado con la ECV. La hostilidad constituye un constructo multidimensional que abarca aspectos cognitivos, fisiológicos, emocionales y conductuales (Donker et al., 2000; Everson-Rose y Lewis, 2005; Matthews et al., 2004). En cuanto a la asociación de la hostilidad con el desarrollo de la $E C V$, distintos estudios han mostrado que la hostilidad, bien puede actuar como un factor de riesgo independiente (Juárez, 2008); o bien puede tener un efecto modulado por otras variables, como la edad, el bajo apoyo social, la hipertensión, la depresión o las conductas de riesgo (Bunde y Suls, 2006; Eaker et al., 2004; Shen, 2002).

Si se considera la dimensión emocional de la hostilidad, se puede decir que ésta puede incluir desde la ligera irritación hasta la ira, pudiendo ser expresada la ira de forma inhibida o manifiesta.

Algunos trabajos (Lampert et al., 2002) han evidenciado que la ira puede constituir un precipitante de isquemia transitoria, y asociarse con un mayor riesgo de sufrir arritmia. En concreto, la ira interiorizada o inhibida ha aparecido relacionada, en personas sanas, con hipertensión, con una baja aserción y depresión, y con el doble de riesgo de sufrir un ataque cardiaco (Everson et al., 1998; Everson et al.,1999). Angerer et al., (2000) señalaron que la interacción entre la expresión de la ira y una baja percepción de apoyo social podría aumentar el riesgo de sufrir una ECV, en mayor medida que si estos dos mismos componentes se presentaban de forma aislada.

Otro estudio realizado desde la perspectiva interpersonal (Smith et al., 2004), indica que la combinación de la dimensión de dominanciasumisión con la ira podría dar lugar a la aparición de diferentes tipos de ECV, utilizando para ello mecanismos distintos. Así por ejemplo, el rasgo de dominancia, junto con la ira expresada de forma directa, podría influir en la aparición de la ECV mediante las respuestas de antagonismo frente los demás. Sin embargo la dominancia acompañada de ira reprimida, podría provocar un aumento en la presión arterial; mientras que la sumisión en combinación con la ira reprimida, podría asociarse con la ECV a través de una mayor necesidad de huida, desconfianza y aislamiento social.

Esta investigación apoya, de algún modo, la línea de otros estudios en los que se pone de manifiesto que las personas hostiles o con un mayor grado de ira, utilizan un estilo de afrontamiento que puede tener consecuencias negativas sobre la ECV.

En algunos estudios se ha observado que los pacientes que padecen algún tipo de ECV, y que se caracterizan por un alto grado de hostilidad, presentan un estilo de afrontamiento menos focalizado en el problema, para afrontar el estrés vital (Sasaki y Kamasaki, 2002); usando otro tipo de estrategias, como la confrontación, la evitación y el distanciamiento o la negación (Vandervoort, 1992, 2006). En la misma dirección se sitúa el estudio de Fontana et al., (1998), en el que se abordó, en un grupo 
de mujeres, la relación entre el estrés psicológico, la reactividad en frecuencia cardiaca y la presión arterial, en función de las estrategias de afrontamiento utilizadas. Los resultados revelaron que acciones como el aislamiento y la baja búsqueda de apoyo social, se relacionaban con niveles más altos en la presión arterial y con el desarrollo de trastornos coronarios.

En otras investigaciones (Shen et al., 2004) se destaca que las estrategias dirigidas a la evitación también podrían ser un factor de riesgo para el desarrollo de la ECV, dada su posible asociación con la elevación en el nivel de reactividad cardiovascular al estrés y con una mayor presión arterial e isquemia coronaria (Fredrikson et al., 2000; Helmers y Krantz, 1996). Gremigni et al. (2000) encontraron que la combinación de una alta hostilidad con un afrontamiento centrado en la evitación de los acontecimientos amenazantes, se vinculaba con un peor estado de salud en personas que habían sido sometidas a un bypass.

Por otra parte hay que mencionar también que la hostilidad representa una característica que puede dañar la calidad de vida en pacientes con ECV. Las personas con un alto grado de hostilidad, no solo responden ante las diferentes situaciones de una forma más desadaptativa, sino que también sus atribuciones y las consecuencias de sus conductas contribuyen a crear un ambiente hostil que aumenta su reactividad fisiológica. Este incremento en la reactividad fisiológica puede dar lugar al deterioro en el funcionamiento cardiovascular, neuroendocrino e inmune, originando más problemas de salud y una disminución en el bienestar global de la persona. (Boyle et al., 2004; Boyle et al., 2006; Boyle et al., 2007; Julkunen y Ahlström, 2006; Lavie y Milani, 2005).

De igual modo la hostilidad puede representar un factor de riesgo en el ámbito de la ECV, en la medida en que propicia la realización de comportamientos de riesgo, tales como fumar, consumir mayor cantidad de alcohol o llevar a cabo un inadecuado seguimiento de la dieta prescrita (Siegler et al., 1992; Vandervoort, 2006).

En términos generales, una conclusión que puede extraerse de la mayoría de los estudios revisados es que, si bien parece que la ira y la depresión ejercen, de forma independiente, un impacto negativo sobre el afrontamiento y la calidad de vida en los pacientes con ECV, no queda claro cuál podría ser el efecto y el peso que tendrían estas dos emociones si se abordaran de forma conjunta. Es por ello que, en la presente investigación, hemos tratado de comparar el peso que tendrían la ira y la depresión en la predicción de los factores de riesgo tradicionales, y en las estrategias de afrontamiento y en la calidad de vida en pacientes con un diagnóstico de ECV.

\section{Objetivos e hipótesis}

En la presente investigación se plantearon los siguientes objetivos:

En primer lugar, se examinó, en una muestra de pacientes cardiovasculares las posibles diferencias en función del sexo, en relación con el tipo de patología cardiovascular padecida, y con determinados factores de riesgo comportamentales, biológicos y psicosociales. Estos últimos incluían las emociones negativas de ira y depresión, las estrategias de afrontamiento y la calidad de vida. 
En segundo lugar se trató de estudiar, de forma conjunta, cómo se relacionaban la ira y la depresión con los factores de riesgo mencionados, las estrategias de afrontamiento y la calidad de vida.

Por último, se pretendió determinar y comparar el peso que tenían la ira y la depresión en la predicción de todos estos criterios.

Por otro lado, teniendo en cuenta los resultados obtenidos en las investigaciones previas, se definieron las siguientes hipótesis: en relación con las diferencias respecto al sexo, se esperaba que las mujeres, en comparación con los hombres presenten un mayor desajuste psicológico ante la ECV; caracterizándose este desajuste por un mayor uso de estrategias de afrontamiento emocionales, por la presencia de un estado emocional más negativo y por una menor calidad de vida. Por otro lado se plantea, como pregunta de investigación, si con respecto a los factores de riesgo biológicos y comportamentales, habrá diferencias significativas en función del sexo.

En cuanto a las posibles relaciones y el peso predictivo de la ira y la depresión con los diferentes factores de riesgo y las variables psicológicas elegidas, se esperaba que ambas emociones se asociarían con la presencia de factores de riesgo comportamentales y con una mayor probabilidad de padecer algún tipo de trastorno cardiovascular. Asimismo se especuló que la ira y la depresión predecirían de forma positiva las estrategias de afrontamiento centradas en la emoción, y de modo negativo la calidad de vida.
Método

Participantes y procedimiento

Con el fin de acceder a los pacientes con patología cardiovascular, se contactó con dos centros sanitarios. Antes de comenzar la investigación se obtuvieron los permisos pertinentes, tanto de la dirección como de los responsables médicos.

Los pacientes que fueron seleccionados para este estudio acudían al centro hospitalario, bien porque se les estaba realizando el seguimiento cardiológico o porque se les había diagnosticado recientemente la enfermedad cardiovascular. Antes de hacerles entrega del cuadernillo con las diferentes escalas, los pacientes fueron informados de la investigación y del carácter voluntario de la misma. Aquellos que presentaban un trastorno psiquiátrico diagnosticado previamente, o unas condiciones físicas que impedían la realización del cuestionario, fueron desestimadas a priori. Inicialmente se accedió a 312 pacientes, los cuales firmaron el consentimiento para participar en el estudio. De estos 312 pacientes, 167 no devolvieron los cuestionarios cumplimentados y 38 tuvieron que ser descartados por haberlo rellenado de forma inadecuada o incompleta.

Entre los pacientes que rellenaron el cuestionario $(n=145)$ y los que no lo hicieron ( $n=$ 167), no se encontraron diferencias significativas con respecto a la edad, el sexo y el tipo de patología padecida.

La muestra final del estudio estuvo compuesta por 107 personas con diferentes problemas cardiovasculares. Un 59,8\% $(n=64)$ eran varones y el $40,2 \%(n=43)$ mujeres. La edad media de la muestra fue de 63 años, siendo el 
rango de edad entre 30 y 87 años. En cuanto al estado civil, un $77,6 \%(n=83)$ de los participantes informó estar casado, y un 22,4 \% (n $=24)$ estar sin pareja. Con respecto al nivel de formación académica, un 41,1\% ( $N=44)$ refirió haber realizado estudios primarios; un 28\% ( $n$ $=30$ ) indicó tener estudios secundarios y bachiller; y un $15 \%(n=16)$ señaló haber cursado estudios universitarios superiores. En relación a la situación laboral un alto porcentaje de personas, un $83,2 \%(n=89)$ se encontraba inactivo, bien por baja temporal o permanente, o bien porque no trabajaban fuera de casa, encontrándose en activo únicamente un 16,8\% $(n=18)$ de los encuestados.

El análisis de los datos se llevó a cabo usando el paquete estadístico SPSS V.15.0.

\section{Instrumentos}

Para realizar el estudio se elaboró un cuadernillo que incluía diferentes escalas relativas a los aspectos sociodemográficos, los hábitos comportamentales, el tipo de enfermedad cardiovascular padecida, los datos biomédicos referidos a los factores de riesgo $y$, por último, las variables psicosociales. Esta información fue suministrada por el propio paciente. Cuando se desconocía alguno de los datos biomédicos, se recurría a la consulta del historial médico.

En particular, entre los aspectos sociodemográficos se recabó información sobre la edad, el sexo, el estado civil (soltero, casado o en pareja, separado o viudo), el nivel de estudios (primarios, secundaria-bachiller, o superiores), y la situación laboral en la que se encontraba el paciente en ese momento.
Respecto a los aspectos comportamentales, los pacientes debían informar si realizaban ejercicio físico, si consumían alcohol, café y tabaco; si llevaban a cabo una dieta cardiosaludable, y si cumplían con las pautas médicas prescritas. El formato de respuesta de estas variables fue de tipo dicotómico (SI-NO).

En relación a los datos biomédicos, los pacientes debían indicar si tenían hipertensión arterial, diabetes u obesidad y si su nivel de triglicéridos, colesterol y azúcar era, respectivamente, elevado. Asimismo se les midió su perímetro abdominal. En lo concerniente al tipo de patología cardiovascular por la que estaban siendo atendidos, se les preguntó si padecían insuficiencia cardiaca, angina de pecho, pericarditis, isquemia coronaria; valvulopatía, problemas del ritmo cardiaco e infarto de miocardio; así como si habían sido sometidos a algún tipo de cirugía, por bypass, cateterismo o trasplante. Asimismo se les pidió que indicaran si tenían antecedentes cardiológicos en familiares de primer grado. El formato de respuesta de todas estas variables fue de tipo dicotómico (SI-NO).

En cuanto a las variables psicosociales, se administraron los siguientes cuestionarios:

Cuestionario Sympton Checklist (SCL-90-R) (Derogatis 1977; adaptación González de Rivera et al., 1989). El SCL-90-R es un inventario que consta de nueve dimensiones, las cuales evalúan: somatización, obsesión-compulsión, sensibilidad interpersonal, depresión, ansiedad, hostilidad, ansiedad fóbica, ideación paranoide y psicoticismo. Entre estas emociones elegimos las relativas a la hostilidad (6 ítems) y la depresión (13 ítems), teniendo en cuenta la relevancia que estas dos emociones han mos- 
trado en el ámbito de la ECV. El formato de respuesta es de tipo Likert (desde $0=$ nada, hasta 4 = muchísimo). La dimensión de hostilidad incluye pensamientos, sentimientos y acciones característicos del estado afectivo negativo caracterizado como rabia, irritabilidad, o ira. Basándonos en estas propiedades, en el estudio presente, hemos optado por utilizar el término ira en lugar del de la hostilidad. La dimensión de depresión, por su parte, recoge signos y síntomas clínicos propios de los trastornos depresivos como el desánimo, la anhedonia, la desesperanza o la falta de energía. El SCL-90-R ha demostrado ser útil, en la medición de la hostilidad en pacientes coronarios (Donker et al., 2000), así como en su aplicación en el ámbito clínico y en el de la investigación (Strik et al., 2001). En el presente estudio, los coeficientes de fiabilidad de las dos subdimensiones estudiadas fueron bastante elevados ( $a=0,83$ para la ira; y $a=0,87$ para la depresión).

Cuestionario Breve de Estrategias de afrontamiento (COPE) (Carver, 1997). Esta versión breve del cuestionario COPE (Carver et al., 1989), está formada por 28 ítem, que miden 14 tipos de reacciones de afrontamiento: afrontamiento activo, planificación, reevaluación positiva, aceptación, humor, religión, uso de apoyo instrumental, uso de apoyo emocional, autodistracción, negación, ventilación emocional, uso de sustancias, desenganche conductual y culpa. Cada subescala consta de 2 ítem, con un formato de respuesta tipo Likert (donde $1=$ no lo hago esto en absoluto, hasta 4 = hago esto muchísimo).

En el presente estudio el contenido de los ítems se refirió a la manera como la persona hacía frente a su ECV o a la cirugía coronaria a la que se hubiera sometido.

El COPE ha sido validado en diferentes áreas de la medicina comportamental, como la relativa al cáncer (Fillion et al., 2002) y a la ECV (Lowe et al., 2000; Whitmarch et al., 2003), demostrando tener buenas propiedades psicométricas, (Murberg y Bru, 2001). En el presente estudio la consistencia interna del total de la escala fue elevada $(a=0,79)$, oscilando los coeficientes de fiabilidad de las distintas subescalas entre 0,42 y 0,89 .

Cuestionario de Calidad de Vida (CCV) (Ruiz y Baca, 1993). Esta escala evalúa el nivel de calidad de vida percibida por la persona. Se compone de 35 preguntas agrupadas en cuatro subáreas: apoyo social (9 ítems), la satisfacción general (13 ítems), el bienestar físico y psicológico (7 ítems), y por último, tiempo libre (6 ítems). El formato de respuesta oscila entre $1=$ nada y $5=$ mucho.

La fiabilidad de la escala global en el presente estudio fue elevada $(a=0,76)$, siendo también altos los coeficientes de fiabilidad para las subdimensiones de apoyo social $(a=0,81)$ y satisfacción general $(a=0,85)$. Sin embargo fueron menores los coeficientes referidos a las subescalas de bienestar $(a=0,46)$ y tiempo libre $(a=0,59)$.

\section{Resultados}

Para examinar las diferencias en función del sexo, se utilizó, en primer lugar, la prueba $t$ para las variables continuas (factores psicosociales), y la prueba $X^{2}$ para las dicotómicas (variables comportamentales, biomédicas y relativas al tipo de patología). 
A continuación se llevó a cabo un análisis correlacional para examinar cómo se asociaban la ira y la depresión con los aspectos comportamentales, biomédicos y relativos al tipo de patología; así como con los factores psicosociales. Con las variables dicotómicas se utilizó la correlación $r_{\mathrm{s}}$ de Spearman y con las cuantitativas la correlación de Pearson.

Finalmente, para comparar el peso de la ira y la depresión, se realizaron análisis de regresión logística, cuando las variable criterio eran dicotómicas; y análisis de regresión lineal múltiple, cuando las variables criterio eran continuas.

\section{Estadísticos descriptivos}

En primer lugar, considerando los hábitos comportamentales (ver Tabla 1), los datos mostraron que un 35\% de la muestra reconocía no hacer nada de ejercicio a la semana. Un 34,6\% informó consumir alcohol, al menos una vez por semana. Un 19,6\% de la muestra indicó ser fumador, habiendo abandonado este hábito con anterioridad un $44,9 \%$ de la muestra. Por último se apreció que más de la mitad de la muestra (un 60\%) no realizaba una dieta saludable, aunque un $83,2 \%$ de los participantes sí manifestó cumplir con el tratamiento médico prescrito.

En cuanto a los factores biomédicos (ver Tabla 2), un $48,6 \%$ de la muestra informó tener niveles elevados de colesterol, y un $56,1 \%$ problemas de hipertensión arterial. El 40,8\% presentó un índice de masa corporal (IMC) mayor de $29,9 \mathrm{~kg} / \mathrm{m}^{2}$, siendo el perímetro abdominal, superior a lo estimado como cardiosaludable, en el 70,4\% de los encuestados. La mayoría de los participantes señalaron no tener problemas relativos a los niveles de triglicéridos (81,3\%), glucemia (86\%) o diabetes (79,4\%). Asimismo, de todos los encuestados, un 51,4\% refirió tener antecedentes familiares de primer grado asociados con la enfermedad cardiaca.

Tabla 1

Descriptivos de las variables comportamentales para la muestra total, y según sexo

\begin{tabular}{|c|c|c|c|}
\hline VARIABLE & $\mathrm{n}(\%)$ & $\begin{array}{l}\text { Hombres } \\
\text { (\%) }\end{array}$ & $\begin{array}{c}\text { Mujeres } \\
(\%)\end{array}$ \\
\hline \multicolumn{4}{|l|}{ EJERCICIO FISICO } \\
\hline Nada & $37(34,6 \%)$ & $19(29,7 \%)$ & $18(41,9 \%)$ \\
\hline Más de $1 \mathrm{vez} / \mathrm{sem}$. & $70(65,4 \%)$ & 45 (70,3\%) & $25(58,1 \%)$ \\
\hline \multicolumn{4}{|l|}{ ALCOHOL } \\
\hline Nada & $70(65,4 \%)$ & $32(50,0 \%)$ & $38(88,4 \%)$ \\
\hline $\begin{array}{l}\text { Si, más de 1vez/ } \\
\text { sem. }\end{array}$ & $37(34,6 \%)$ & $32(50,0 \%)$ & $5(11,6 \%)$ \\
\hline \multicolumn{4}{|l|}{ CAFEINA } \\
\hline No & $53(49,5 \%)$ & $28(43,8 \%)$ & $25(58,1 \%)$ \\
\hline $\begin{array}{l}\text { Si, más de una vez/ } \\
\text { sem. }\end{array}$ & $54(50,5 \%)$ & $36(56,3 \%)$ & $18(41,9 \%)$ \\
\hline \multicolumn{4}{|l|}{ TABACO } \\
\hline Nunca fumó & $38(35,5 \%)$ & $10(15,6 \%)$ & $28(65,1 \%)$ \\
\hline Lo ha dejado & $48(44,9 \%)$ & $39(60,9 \%)$ & $9(20,9 \%)$ \\
\hline Sigue fumando & $21(19,6 \%)$ & $15(23,4 \%)$ & $6(14,0 \%)$ \\
\hline \multicolumn{4}{|l|}{$\begin{array}{l}\text { DIETA } \\
\text { cardiosaludable }\end{array}$} \\
\hline No (nada o poco) & $64(59,8 \%)$ & $40(62,5 \%)$ & $24(55,5 \%)$ \\
\hline $\begin{array}{l}\text { Si (bastante o } \\
\text { mucho) }\end{array}$ & $43(40,2 \%)$ & $24(37,5 \%)$ & $19(44,2 \%)$ \\
\hline \multicolumn{4}{|l|}{$\begin{array}{l}\text { CUMPLIMIENTO } \\
\text { PAUTAS }\end{array}$} \\
\hline No (nada o poco) & $8(16,8 \%)$ & $10(15,6 \%)$ & $8(18,6 \%)$ \\
\hline $\begin{array}{l}\text { Si (bastante o } \\
\text { mucho) }\end{array}$ & $89(83,2 \%)$ & $54(84,4 \%)$ & $35(81,4 \%)$ \\
\hline
\end{tabular}

En lo que concierne a la patología cardiaca padecida (ver Tabla 2), el infarto de miocardio (IM), así como los trastornos cardiovasculares (TCV), los cuales incluían la insuficiencia cardiaca, la pericarditis, angina de pecho y la cardiopatía isquémica, representaron las causas principales por las que los participantes 
Tabla 2

Descriptivos de las variables biomédicas para la muestra total, y según sexo

\begin{tabular}{|c|c|c|c|}
\hline VARIABLE & n (\%) & Hombres (\%) & Mujeres (\%) \\
\hline \multicolumn{4}{|l|}{ TRIGLICÉRIDOS } \\
\hline $\mathrm{No} \leq 160 \mathrm{mg} / \mathrm{dl}$ & $87(81,3 \%)$ & $54(84,4 \%)$ & $33(76,7 \%)$ \\
\hline $\mathrm{Si}>160 \mathrm{mg} / \mathrm{dl}$ & $20(18,7 \%)$ & $10(15,6 \%)$ & $10(23,3 \%)$ \\
\hline \multicolumn{4}{|l|}{ GLUCEMIA } \\
\hline $\mathrm{No} \leq 126 \mathrm{mg} / \mathrm{dl}$ & $92(86,0 \%)$ & $56(87,5 \%)$ & $36(83,7 \%)$ \\
\hline $\mathrm{Si}>126 \mathrm{mg} / \mathrm{dl}$ & $15(14,0 \%)$ & $8(12,5 \%)$ & $7(16,3 \%)$ \\
\hline \multicolumn{4}{|l|}{ DIABETES } \\
\hline No & $85(79,4 \%)$ & $50(78,1 \%)$ & $35(81,4 \%)$ \\
\hline $\mathrm{Si}$ & $22(20,6 \%)$ & $14(21,9 \%)$ & $8(18,6 \%)$ \\
\hline \multicolumn{4}{|l|}{ COLESTEROL } \\
\hline $\mathrm{No} \leq 220 \mathrm{mg} / \mathrm{dl}$ & $55(51,4 \%)$ & $33(51,6 \%)$ & $22(51,2 \%)$ \\
\hline $\mathrm{Si}>220 \mathrm{mg} / \mathrm{dl}$ & $52(48,6 \%)$ & $31(48,4 \%)$ & $21(48,8 \%)$ \\
\hline \multicolumn{4}{|l|}{ HIPERTENSIÓN ARTERIAL } \\
\hline No $\leq 13,5 \mathrm{mmhg} ; 8,5 \mathrm{mmhg}$ & $47(43,9 \%)$ & $30(46,9 \%)$ & $17(39,5 \%)$ \\
\hline $\mathrm{Si}>13,5 \mathrm{mmhg} ; 8,5 \mathrm{mmhg}$ & $60(56,1 \%)$ & $34(53,1 \%)$ & $26(60,5 \%)$ \\
\hline \multicolumn{4}{|l|}{ IMC (Indice masa corporal) } \\
\hline $\mathrm{No} \leq 29,9 \mathrm{~kg} . / \mathrm{m}^{2}$ & $64(59,2 \%)$ & $40(62,5 \%)$ & $24(55,8 \%)$ \\
\hline $\mathrm{Si}>29,9 \mathrm{~kg} . / \mathrm{m}^{2}$ & $43(40,8 \%)$ & $24(37,5 \%)$ & $19(44,2 \%)$ \\
\hline \multicolumn{4}{|l|}{ PERIMETRO CINTURA } \\
\hline No & $31(29,6 \%)$ & $17(26,6 \%)$ & $14(32,6 \%)$ \\
\hline $\mathrm{Si}>88$ mujer, $>94$ hombre & $76(70,4 \%)$ & $47(73,4 \%)$ & $29(67,4 \%)$ \\
\hline \multicolumn{4}{|l|}{ ANTECEDENTES FAMILIARES } \\
\hline No & $52(48,6 \%)$ & $36(56,3 \%)$ & $16(37,2 \%)$ \\
\hline Si (familiar $1^{\circ}$ grado) & $55(51,4 \%)$ & $28(43,6 \%)$ & $27(62,8 \%)$ \\
\hline \multicolumn{4}{|l|}{ TIPO DE CARDIOPATÍA** } \\
\hline T.Cardiovascular* & $49(45,8 \%)$ & $23(36,0 \%)$ & $26(60,4 \%)$ \\
\hline Cirugía (bypass/cateter) & $28(26,1 \%)$ & $18(28,1 \%)$ & $10(23,3 \%)$ \\
\hline Infarto agudo miocardio & $43(40,2 \%)$ & $32(50,0 \%)$ & $11(25,6 \%)$ \\
\hline Valvulopatía & $6(5,6 \%)$ & $2(3,1 \%)$ & $4(9,3 \%)$ \\
\hline Fibrilación & $13(12,1 \%)$ & $9(14,1 \%)$ & $4(9,3 \%)$ \\
\hline Arritmias & $27(25,2 \%)$ & $15(23,4 \%)$ & $12(27,9 \%)$ \\
\hline
\end{tabular}

* insuficiencia cardiaca, pericarditis, cardiopatía isquémica o angina de pecho

** los porcentajes descritos se refieren a las personas que sí padecen esa patología 
estaban siendo asistidos en el servicio de cardiología (un 40,2\% presentaba IM y un 45,8\% algún otro tipo de TCV). La distribución de las frecuencias del resto de patologías (valvulopatía, fibrilación y/o arritmia) fue notablemente inferior.
$0,82)$ como en depresión $(M=1,04)$ unas puntuaciones medias superiores a las alcanzadas en población general $(M=0,45$ para la ira; $M=0,7$ para la depresión [Derogatis, 1977]) y similares a las medias en población con patología temporomandibular $(M=0,82$ para la ira; $M=1,14$ para

Tabla 3

Descriptivos de las variables psicológicas para la muestra total, y según sexo

\begin{tabular}{|c|c|c|c|c|c|}
\hline & & & & & \\
\hline & $\begin{array}{c}\text { Muestra Total } \\
\text { Media } \pm \text { DT }\end{array}$ & Mínimo & Máximo & $\begin{array}{l}\text { Hombres } \\
\text { Media } \pm \text { DT }\end{array}$ & $\begin{array}{c}\text { Mujeres } \\
\text { Media } \pm \text { DT }\end{array}$ \\
\hline \multicolumn{6}{|l|}{ Emociones } \\
\hline Ira & $0,82 \pm 0,88$ & 0 & 6 & $0,89 \pm 0,98$ & $0,70 \pm 0,73$ \\
\hline Depresión & $1,04 \pm 0,71$ & 0,7 & 3,08 & $0,89 \pm 0,57$ & $1,27 \pm 0,84$ \\
\hline \multicolumn{6}{|l|}{ Estrategias de afrontamiento } \\
\hline Afrontamiento Activo & $5,12 \pm 1,29$ & 2 & 8 & $5,1 \pm 1,31$ & $5,09 \pm 1,27$ \\
\hline Planificación & $4,82 \pm 1,53$ & 2 & 8 & $4,6 \pm 1,49$ & $5,07 \pm 1,58$ \\
\hline Reestructuración Positiva & $4,67 \pm 1,59$ & 2 & 8 & $4,87 \pm 1,45$ & $4,37 \pm 1,75$ \\
\hline Aceptación & $5,28 \pm 1,56$ & 2 & 8 & $5,2 \pm 1,44$ & $5,35 \pm 1,73$ \\
\hline Humor & $3,47 \pm 1,59$ & 2 & 8 & $3,40 \pm 1,48$ & $3,58 \pm 1,75$ \\
\hline Religión & $4,10 \pm 2,09$ & 2 & 8 & $3,64 \pm 1,89$ & $4,84 \pm 2,19$ \\
\hline Uso Apoyo Emocional & $5,09 \pm 1,57$ & 2 & 8 & $5,26 \pm 1,45$ & $4,83 \pm 1,73$ \\
\hline Uso Apoyo Instrumental & $4,63 \pm 1,51$ & 2 & 8 & $4,71 \pm 1,37$ & $4,51 \pm 1,71$ \\
\hline Autodistracción & $4,29 \pm 1,82$ & 2 & 8 & $4,11 \pm 1,67$ & $4,56 \pm 2,01$ \\
\hline Negación & $3,53 \pm 1,54$ & 2 & 8 & $3,43 \pm 1,53$ & $3,67 \pm 1,57$ \\
\hline Ventilación Emocional & $4,39 \pm 1,77$ & 2 & 8 & $4,33 \pm 1,60$ & $4,49 \pm 2,00$ \\
\hline Uso Sustancias & $2,11 \pm 0,63$ & 2 & 7 & $2,14 \pm 0,73$ & $2,07 \pm 0,45$ \\
\hline Desenganche Conductual & $3,44 \pm 1,34$ & 2 & 8 & $3,40 \pm 1,41$ & $3,49 \pm 1,26$ \\
\hline Culpa & $4,17 \pm 1,75$ & 2 & 8 & $4,07 \pm 1,39$ & $4,30 \pm 2,18$ \\
\hline \multicolumn{6}{|l|}{ Calidad de vida } \\
\hline Apoyo Social & $34,45 \pm 5,49$ & 13 & 45 & $34,95 \pm 4,9$ & $33,72 \pm 6,11$ \\
\hline Satisfacción general & $44,39 \pm 9,25$ & 15 & 65 & $45,84 \pm 8,38$ & $42,23 \pm 10,13$ \\
\hline $\begin{array}{l}\text { Bienestar físico y } \\
\text { psicológico }\end{array}$ & $22,55 \pm 5,89$ & 7 & 35 & $23,37 \pm 5,68$ & $21,32 \pm 6,06$ \\
\hline Tiempo Libre & $21,38 \pm 4,64$ & 9 & 30 & $21,50 \pm 4,66$ & $21,20 \pm 4,65$ \\
\hline Total Calidad de Vida & $123,11 \pm 18,30$ & 57 & 158 & $126,04 \pm 16,87$ & $118,7 \pm 19,64$ \\
\hline
\end{tabular}

Los estadísticos descriptivos relativos a las emociones de la ira y la depresión, las estrategias de afrontamiento y la calidad de vida se muestran en la Tabla 3. Como se observa en dicha tabla, la depresión fue el estado negativo más frecuente. Asimismo, los pacientes de nuestro estudio obtuvieron tanto en ira $(M=$ la depresión [Derogatis, 1977]). Asimismo, los niveles medios en ira y depresión alcanzados en este estudio fueron algo menores a los referidos en población psiquiátrica $(M=1,18$ para la ira y $M=1,61$ para la depresión [Derogatis, 1977]).

En cuanto a las estrategias de afrontamiento, las más utilizadas fueron las referidas al afronta- 
miento activo, la planificación, el uso de apoyo tanto emocional como instrumental y la aceptación. Las menos usadas fueron las relativas al uso de sustancias, el desenganche conductual, la negación y el sentido del humor. Estos datos son similares a los mostrados por otros estudios como el de Murberg y Bru (2001).

Por último, con respecto a los estadísticos relativos a la calidad de vida, nuestros resultados reflejaron unas puntuaciones más bajas, tanto en la escala global como en todas las subdimensiones, en comparación con las medias obtenidas para la población general (Ruiz y Baca, 1993). Las puntuaciones medias de nuestro estudio fueron también ligeramente superiores a las medias en población psiquiátrica (Ruiz y Baca, 1993).

\section{Diferencias de sexo}

Con respecto a los factores comportamentales evaluados, se observó que había algunas diferencias significativas en función del sexo.

Por una parte, el porcentaje de hombres (50\%) que consumía alcohol era significativamente más alto que el de las mujeres (11,6\%; $\left.X_{(1)}^{2}=16,74, p<0,001\right)$. Entre las personas fumadoras, el porcentaje de hombres que continuaba fumando (23,45\%) era también más elevado que el de las mujeres (14\%), $\left(X_{(2)}^{2}=28,09, p<\right.$ $0,000)$. Además, un $62,8 \%$ de las mujeres declaró tener antecedentes familiares de primer grado, mientras que, en el caso de los hombres, la presencia de antecedentes sólo se dio en el 43,6\%, siendo la diferencia entre ambos grupos marginalmente significativa $\left(X_{(1)}^{2}=3,73, p=0,05\right)$.

En cuanto al resto de los factores comportamentales y la totalidad de los factores bio- médicos, no se encontraron diferencias significativas.

Atendiendo a la patología cardiaca se constató que los trastornos cardiovasculares eran más frecuentes en las mujeres que en los hombres. En concreto, un $60,4 \%$ de las mujeres de la muestra refirió tener, al menos, uno de los trastornos incluidos en esa categoría, mientras que en el caso de los hombres, este porcentaje fue menor (36\%), $\left(x_{(1)}^{2}=6,23, p<0,05\right)$. No obstante, el porcentaje de hombres (50\%) que indicó haber sufrido un infarto de miocardio, fue significativamente superior al porcentaje de mujeres $(25,6 \%)$ con la misma condición $\left(X_{(1)}^{2}=6,38, p<0,05\right)$. Con respecto al resto de las cardiopatías analizadas (cirugía, arritmia, valvulopatía o fibrilación) no se encontraron diferencias en función de sexo.

En lo referente a las emociones negativas, las mujeres presentaron más síntomas depresivos $\left(t_{(67)}=-2,62, p<0,05\right)$ que los hombres, si bien no de encontraron diferencias entre ambos grupos en relación con la ira. Tampoco hubo diferencias significativas en la utilización de la mayor parte de estrategias de afrontamiento, salvo para la estrategia "religión" que sí fue más usada por las mujeres $\left(t_{(105)}=-3,004 ; p\right.$ $<0,005)$. Por último, no se apreciaron diferencias en la calidad de vida global, en el apoyo social, bienestar y en el tiempo libre; aunque las mujeres refirieron tener una satisfacción general más baja que los hombres $\left(t_{(105)}=2,01\right.$; $p<0,05)$.

En resumen, respecto a las diferencias en cuanto al sexo, los resultados obtenidos pusieron de manifiesto que los hombres consumían mayor cantidad de alcohol y tabaco, e 
informaban, en mayor medida, haber sufrido infarto de miocardio. En contraste, la presencia de otro tipo de trastornos cardiovasculares, diferentes al infarto de miocardio, fue más frecuente en las mujeres. Estas, además, indicaron utilizar con mayor frecuencia la estrategia de la religión para afrontar su enfermedad; y refirieron niveles más elevados de depresión y una satisfacción personal más baja.

Correlaciones de la ira y la depresión con el resto de variables

Tabla 4

Correlaciones de la ira y depresión con los factores de riesgo comportamentales y biológicos

\begin{tabular}{lcc}
\cline { 2 - 3 } & IRA & DEPRESIÓN \\
\hline Ejercicio físico & 0,03 & $-0,13$ \\
Tabaco & 0,16 & $-0,07$ \\
Consumo de café & 0,92 & $-0,04$ \\
Consumo de alcohol & 0,07 & $-0,17$ \\
Dieta & 0,09 & $0,22^{*}$ \\
Cumplimiento & $-0,20^{*}$ & $-0,07$ \\
Triglicéridos & $-0,12$ & $-0,05$ \\
Glucemia & $-0,01$ & $-0,01$ \\
Diabetes & $-0,04$ & $-0,02$ \\
Colesterol & 0,03 & 0,03 \\
HTA & 0,05 & $-0,02$ \\
IMC & $0,21^{*}$ & 0,12 \\
Perímetro & 0,17 & 0,18 \\
Antecedente familiar & 0,06 & 0,15 \\
ECV & 0,11 & $0,25^{*}$ \\
Cirugía & $-0,07$ & $-0,05$ \\
Infarto de miocardio & $-0,06$ & $-0,13$ \\
Valvulopatía & 0,09 & 0,18 \\
Fibrilación & $-0,01$ & 0,09 \\
Arritmias & 0,16 & 0,09 \\
\hline
\end{tabular}

${ }^{*} p<0,05$

Las correlaciones de la ira y la depresión con los diferentes factores de riesgo comportamentales, biomédicos, y cardiopatológicos apenas fueron significativas (ver Tabla 4). No obstante, sí destacó la asociación significativa de la ira con un menor cumplimiento de las prescripciones médicas, y con un índice de masa corporal más alto. Asimismo la depresión correlacionó con un mayor seguimiento de la dieta y con el hecho de padecer ECV.

Tabla 5

Correlaciones de la ira y la depresión con las estrategias de afrontamiento y calidad de vida

\begin{tabular}{lcc} 
& IRA & DEPRESIÓN \\
\hline Afrontamiento activo & 0,05 & 0,06 \\
Planificación & $-0,02$ & 0,13 \\
Reestructuración & $-0,06$ & $-0,08$ \\
positiva & & \\
Aceptación & $-0,07$ & $-0,08$ \\
Uso de apoyo emocional & $-0,03$ & 0,04 \\
Uso apoyo Instrumental & $0,19^{*}$ & 0,16 \\
Humor & 0,01 & $-0,07$ \\
Religión & $-0,09$ & 0,06 \\
Autodistracción & $0,33^{* * *}$ & $0,38^{* * *}$ \\
Negación & $0,21^{*}$ & $0,37^{* * *}$ \\
Ventilación emocional & $0,29^{* *}$ & $0,22^{*}$ \\
Desenganche conductal & 0,17 & $0,25^{* *}$ \\
Culpa & $0,20^{*}$ & $0,43^{* * *}$ \\
Calidad Vida Total & $-0,29^{* *}$ & $-0,46^{* * *}$ \\
Apoyo social & $-0,08$ & $-0,18$ \\
Satisfacción & $-0,13$ & $-0,36^{* * *}$ \\
Bienestar & $-0,31^{* * *}$ & $-0,46^{* * *}$ \\
Tiempo libre & $-0,30^{* * *}$ & $-0,24^{*}$ \\
\hline
\end{tabular}

${ }^{* * *} p<0,001 \quad{ }^{* *} p<0,01 \quad{ }^{*} p<0,05$

Acerca de la relación de la ira y la depresión con las estrategias de afrontamiento, encontramos que tanto la ira como la depresión correlacionaban de forma positiva con las estrategias de autodistracción, negación, ventilación emocional y culpa. La ira, a su vez, se relacionó con un mayor uso de la estrategia de apoyo instrumental; mientras que la depresión lo hizo con el desenganche conductual. Con el resto de estrategias, las correlaciones obteni- 
das no resultaron significativas (ver Tabla 5).

Por último, se observó que tanto la ira como la depresión se asociaban con una menor calidad de vida total y con un menor nivel de bienestar y tiempo libre. La depresión, además, se relacionó con un grado de satisfacción más bajo. Ninguna de las dos emociones mostró una asociación significativa con el apoyo social (ver Tabla 5). tilación emocional y culpa que llevaban a cabo los pacientes. Además, estos dos estados afectivos se relacionaron con una calidad de vida más baja, con menos bienestar físico y psicológico, y con una menor disponibilidad de tiempo libre. En el caso de la depresión, hay que mencionar también su relación con una disminución en el grado de satisfacción general.

\section{Tabla 6.}

Predicción de los factores de riesgo comportamentales y del tipo de patología cardiovascular, a partir de la ira y la depresión

\begin{tabular}{|c|c|c|c|c|c|c|c|c|}
\hline & \multicolumn{3}{|c|}{ Ira } & \multicolumn{5}{|c|}{ Depresión } \\
\hline & B & Sig. & $\operatorname{Exp}(B)$ & C.I. (95\%) & B & Sig. & $\operatorname{Exp}(ß)$ & C.I. (95\%) \\
\hline Alcohol & 0,435 & 0,135 & 1,545 & $\begin{array}{r}0,87 \\
2,7\end{array}$ & $-1,033^{*}$ & 0,018 & 0,356 & $\begin{array}{l}0,15 \\
0,83\end{array}$ \\
\hline Tabaco & $0,809^{*}$ & 0,015 & 2,245 & $\begin{array}{l}1,17 \\
4,31\end{array}$ & $-0,929$ & $0,072^{\#}$ & 0,395 & $\begin{array}{l}0,14 \\
1,08\end{array}$ \\
\hline Dieta & $-0,401$ & 0,168 & 0,669 & $\begin{array}{l}0,37 \\
1,18\end{array}$ & $0,993^{* *}$ & 0,008 & 2,698 & $\begin{array}{l}1,29 \\
5,61\end{array}$ \\
\hline ECV & $-0,486$ & 0,097 & 0,615 & $\begin{array}{l}0,34 \\
1,09\end{array}$ & $1,040^{* *}$ & 0,007 & 2,829 & $\begin{array}{l}1,33 \\
5,99\end{array}$ \\
\hline
\end{tabular}

${ }^{* *} p<0,01 \quad{ }^{*} p<0,05 \quad \# p=0,07$

Por lo tanto, basándonos en estos resultados, se pudo apreciar que la ira se asociaba con indicadores biomédicos y comportamentales más negativos, como eran un mayor índice de masa corporal y un seguimiento más bajo de las prescripciones médicas, respectivamente. La depresión, por el contrario, se relacionó con un mayor seguimiento de la dieta y también con la presencia de algún tipo de trastorno cardiovascular. Por otra parte, tanto la ira como la depresión se asociaron con el empleo de estrategias de afrontamiento centradas principalmente en la emoción, aspecto este que quedó reflejado en las respuestas de distracción, negación, ven-
Predicción de los factores de riesgo comportamentales y biomédicos, y del tipo de patología cardiovascular

A continuación realizamos diferentes análisis de regresión logística, para determinar el valor predictivo de la ira y la depresión, en relación con los factores de riesgo comportamentales, y biomédicos, y con la patología cardiovascular. Dichos análisis (ver Tabla 6) mostraron que la depresión se asociaba con un mayor riesgo de padecer ECV $(\operatorname{Exp}(ß)=2,829$, $p<0,01)$, con una mayor probabilidad de seguir la dieta $(\operatorname{Exp}(B)=2,698, p<0,01)$ y con un riesgo más bajo de consumir alcohol $(\operatorname{Exp}(ß)=$ 
$0,356, p<0,05)$ y de fumar $(\operatorname{Exp}(ß)=0,395, p$ $<0,07)$, si bien en este último caso la significación obtenida fue marginal. La ira, por su parte, apareció sólo asociada con un mayor riesgo de fumar $(\operatorname{Exp}(\beta)=2,245, p<0,05)$. En cuanto al resto de factores estudiados, no se encontró ninguna asociación significativa.

Predicción de las estrategias de afrontamiento y la calidad de vida

Tabla 7

Predicción de las estrategias de afrontamiento a partir de la ira y la depresión

\begin{tabular}{|c|c|c|c|c|}
\hline & $\mathrm{R}^{2}$ & $\begin{array}{c}\text { Cambio } \\
\mathrm{R}^{2} \\
\end{array}$ & $F_{(2,106)}$ & B \\
\hline \multicolumn{5}{|l|}{$\begin{array}{l}\text { Uso apoyo } \\
\text { instrumental }\end{array}$} \\
\hline IRA & 0,037 & 0,037 & $2,21^{*}$ & 0,15 \\
\hline DEPRESIÓN & 0,04 & 0,004 & & 0,08 \\
\hline \multicolumn{5}{|l|}{ Autodistracción } \\
\hline IRA & 0,11 & 0,11 & $10,21^{* * *}$ & 0,18 \\
\hline DEPRESIÓN & 0,16 & 0,05 & & $0,27^{*}$ \\
\hline \multicolumn{5}{|l|}{ Negación } \\
\hline IRA & 0,04 & 0,04 & $8,30 * * *$ & 0,002 \\
\hline DEPRESIÓN & 0,14 & 0,09 & & $0,37^{* * *}$ \\
\hline \multicolumn{5}{|l|}{$\begin{array}{l}\text { Ventilación } \\
\text { emocional }\end{array}$} \\
\hline IRA & 0,09 & 0,09 & $5,235^{* *}$ & $0,246^{*}$ \\
\hline DEPRESIÓN & 0,09 & 0,00 & & 0,086 \\
\hline \multicolumn{5}{|c|}{$\begin{array}{l}\text { Desenganche } \\
\text { conductal }\end{array}$} \\
\hline IRA & 0,03 & 0,03 & $3,48^{* *}$ & 0,041 \\
\hline DEPRESIÓN & 0,06 & 0,04 & & $0,225^{\Delta}$ \\
\hline \multicolumn{5}{|l|}{ Culpa } \\
\hline IRA & 0,04 & 0,04 & $11,78^{* * *}$ & $-0,06$ \\
\hline DEPRESIÓN & 0,18 & 0,15 & & $0,46^{* * *}$ \\
\hline
\end{tabular}

${ }^{* * *} p<0,001 \quad{ }^{* *} p<0,01{ }^{*} p<0,05 \quad{ }^{\Delta} p=0,053$

Por último se trató de determinar, cómo la ira y la depresión contribuían a explicar el tipo de estrategias de afrontamiento que utilizaban los pacientes cardíacos ante su problema de salud, y su calidad de vida. En cuanto a la predicción del afrontamiento, sólo seleccionamos, como variables criterio, las estrategias de afrontamiento que previamente habían correlacionado de forma significativa con la ira y la depresión.

Tabla 8

Predicción de la calidad de vida a partir de la ira y la depresión

$\begin{array}{lccc}R^{2} & \begin{array}{c}\text { Cambio } \\ R^{2}\end{array} & F_{(2,106)} & B\end{array}$

\section{Satisfacción}

$\begin{array}{llllr}\text { IRA } & 0,02 & 0,02 & 8,098^{* * *} & 0,101 \\ \text { DEPRESIÓN } & 0,13 & 0,12 & & -0,414^{* * *}\end{array}$

Bienestar

$\begin{array}{llllr}\text { IRA } & 0,098 & 0,098 & 14,16^{* * *} & -0,08 \\ \text { DEPRESIÓN } & 0,214 & 0,116 & & -0,413^{* * *}\end{array}$

Tiempo libre

IRA $\quad 0,090 \quad 0,090 \quad 5,556^{* *} \quad-0,243^{*}$

DEPRESIÓN $\quad 0,097 \quad 0,007 \quad-0,101$

Calidad de

vida total

\begin{tabular}{llllr} 
IRA & 0,082 & 0,082 & $13,826^{* * *}$ & $-0,043$ \\
DEPRESIÓN & 0,210 & 0,128 & & $-0,433^{* * *}$ \\
\hline
\end{tabular}

${ }^{* * *} p<0,001{ }^{* *} p<0,01{ }^{*} p<0,05$

La depresión contribuyó a explicar las estrategias de autodistracción ( $(=0,27 ; p<$ $0,05)$, negación $(B=0,37 ; p<0,001)$, y desenganche conductual ( $\beta=0,22 ; p=0,05)$; además de asociarse con un aumento en la culpabilización ( $ß=0,46 ; p<0,001)$. La ira, por su parte, predijo de forma positiva el uso de la ventilación emocional $(\beta=0,246 ; p<0,05)$. En relación con el apoyo instrumental, ninguna de estas dos emociones tuvo un peso explicativo (ver Tabla 7).

Con respecto a la contribución de la ira y la depresión sobre la calidad de vida y sus dife- 
rentes facetas, los análisis de regresión indicaron que la depresión se asociaba, de forma significativa, con una disminución en la calidad de vida total $(\beta=-0,43 ; p<0,001)$, en el nivel de satisfacción $(B=-0,414 ; p<0,001)$ y en el grado de bienestar físico y psicológico $(\beta$ $=-0,41 ; p<0,001)$. La ira, sin embargo, sólo predijo de forma negativa el tiempo libre ( $\beta=$ $-0,243 ; p<0,05)$. En cuanto a la predicción del apoyo social, ni la ira ni la depresión resultaron significativa (ver Tabla 8).

\section{Discusión}

El presente estudio ha pretendido abordar dos cuestiones relevantes dentro del ámbito de la ECV. En primer lugar hemos examinado, en una muestra de pacientes cardiológicos, las posibles diferencias de sexo con respecto a determinados factores de riesgo, el tipo de problema cardiovascular padecido, y diversos aspectos psicosociales. En particular se ha considerado las emociones de la ira y la depresión, las estrategias de afrontamiento y la calidad de vida. En segundo lugar se ha estudiado, en relación con estos criterios cardiovasculares y psicosociales, cuál era la asociación y el peso predictivo de la ira y la depresión.

En cuanto a las diferencias de sexo, los resultados obtenidos pusieron de manifiesto que los pacientes varones consumían más cantidad de alcohol y tabaco que las mujeres e informaban, en mayor medida, haber sufrido un infarto de miocardio.

Las mujeres, en cambio, referían haber padecido en mayor grado otro tipo de ECV diferente al infarto, como la insuficiencia cardiaca, la angina de pecho, pericarditis o la isquemia coronaria.
De igual forma las mujeres indicaron utilizar con mayor frecuencia la estrategia de la religión para afrontar su problema cardiovascular y presentaron un nivel de depresión más elevado y un grado de satisfacción personal más bajo.

Estos resultados coinciden con los aportados por otros estudios (Abbey y Stewart, 2000; Boutin-Foster et al., 2007; Bute et al., 2003; Cahrart y Ades, 1998; Grace et al., 2002), en los que se aprecia que las mujeres con ECV presentan, por lo general, un perfil de vulnerabilidad física y psicológica superior al de los hombres. Este perfil de vulnerabilidad psicológica queda plasmado en nuestro estudio a través de la comorbilidad psiquiátrica que caracteriza a las mujeres, en las que la presencia del estado depresivo es más frecuente.

Se podría apuntar que la asunción del rol femenino tradicional, basado en el cuidado de los demás y en una falta de atención hacia las propias necesidades, podría estar influyendo en el hecho de que las mujeres con ECV dedicaran menos esfuerzo al cuidado de su salud o se involucraran menos en los programas de rehabilitación cardiovascular (Bromberger y Matthews, 1996; Goodwin y Gotlib, 2004; Leong et al., 2004; Lin et al., 2008).

No obstante hay otros elementos que, en este sentido deben tenerse en cuenta, como la edad media de las mujeres que formaron parte del estudio (media $=64$ años), así como el hecho de que, después del climaterio, aparece en las mujeres un mayor número de complicaciones biológicas, como la hipertensión y la obesidad. Estos factores podrían estar asociados, en la mujer, con un mayor riesgo de presentar diferentes tipos de ECV, y con una peor adaptación a la enfermedad, 
una vez que ésta se diagnostica (Bromberger y Matthews, 1996; Lin et al., 2008).

En el caso de los pacientes varones destacó, sin embargo, el caracterizarse por tener más factores de riesgo de tipo comportamental, como consumir con mayor frecuencia alcohol y tabaco, y el presentar, en mayor grado, un diagnóstico de infarto de miocardio. Estos resultados van en la misma dirección que los ofrecidos por investigaciones previas (Weidner, 2000) y son congruentes con la posibilidad de que, tanto el tabaco como el alcohol, podrían estar incrementando, en el grupo de hombres, el riesgo de padecer un infarto de miocardio.

Con respecto a las relaciones de la ira y la depresión con los diferentes factores de riesgo biológicos y comportamentales, los datos correlacionales revelaron que la ira se asociaba con una peor adherencia a las prescripciones médicas y con un IMC más elevado. Sin embargo, en los modelos de regresión logística, se asoció sólo con un mayor riesgo de consumir tabaco. La depresión, en cambio, correlacionó con un consumo de alcohol más bajo. Pero, en los análisis de regresión, se asoció también con una mayor probabilidad de seguir una dieta saludable, y de padecer algún tipo de trastorno cardiovascular, tales como la insuficiencia cardiaca, la cadiopatía isquémica o la angina de pecho.

Estos resultados, por una parte, son relevantes porque apoyan, de forma empírica, una importante línea de investigación que avala el papel que puede tener la depresión en el inicio de la ECV (Davidson et al., 2006; Frasure-Smith y Lésperance, 2005, 2008; Penninx et al., 2001).

Pero, por otra parte, contrastan con los reflejados en otros estudios, en los que se demuestra que la presencia de la depresión se relaciona con la falta de adherencia a los tratamientos y con un bajo cumplimiento de las conductas saludables (Fenton y Stover, 2006; Ibishi et al., 2009). Una variable que podría estar influyendo en este sentido en nuestro estudio, es el posible consumo, por parte de los participantes, de fármacos antidepresivos. Este consumo resulta incompatible, en la mayoría de los casos, con el consumo de alcohol. Además es posible que, puesto que muchos antidepresivos poseen efectos secundarios, tales como la retención de líquidos y el aumento de peso, los pacientes del estudio que estuvieran tomando medicación antidepresiva, también podrían estar intentando controlar ese incremento de peso llevando a cabo una dieta más saludable.

Con respecto al papel de la ira y la depresión en la predicción de las estrategias de afrontamiento que utilizaban los pacientes para manejar su enfermedad, se observa que la depresión predecía, en gran medida, el uso de estrategias centradas en la emoción. En particular, la depresión se asoció positivamente con la autodistracción, el desenganche conductual, la negación y la culpabilidad. La ira tuvo un peso bastante menor en la explicación de las diferentes estrategias de afrontamiento, ya que sólo emergió como un predictor positivo y significativo de la ventilación emocional.

Estos resultados, consistentes con investigaciones previas (Cameron, 2009; Vandervoort, 2006), vienen a señalar que, en los enfermos coronarios, la ocurrencia de estados emocionales negativos como la ira y, particularmente, la depresión promueven un afrontamiento más desadaptativo de la enfermedad, dando lugar a con- 
ductas más ineficaces en lo que a la regulación emocional se refiere.

Por otra parte resulta interesante constatar que ni la ira ni la depresión se asociaron de forma negativa con otras estrategias de afrontamiento que podrían considerarse más adaptativas, como la planificación de soluciones o la reevaluación positiva (Zeidner y Saklofske, 1996). Este dato dejaría abierta la posibilidad de considerar si el uso de esas estrategias, más ajustadas a la realidad y por tanto más exitosas en sus resultados, podrían estar determinadas por la presencia de emociones más positivas e independientes de las negativas. Esta idea, aunque tentativa, merece ser considerada en futuros estudios, por su potencial relevancia para detectar, desde el punto de vista emocional, los posibles recursos y limitaciones con los que cuenta el paciente a la hora de manejar su enfermedad.

Finalmente los datos de este estudio revelaron que la presencia de la depresión también afectaba a la calidad de vida de los pacientes, disminuyendo tanto su nivel global, como el grado de satisfacción y bienestar experimentados. La ira, por su parte, sólo se relacionó con la dimensión del tiempo libre, aunque también predijo este criterio de forma negativa.

De este modo se puede decir que la presencia de la ira y la depresión, además de tener consecuencias negativas sobre la forma específica como los pacientes coronarios responden ante su enfermedad, tiende a deteriorar aspectos más generalizados de la esfera del paciente, como son su calidad de vida global, su satisfacción personal y su bienestar.

Con respecto al apoyo social pudimos apreciar que ni la ira ni la depresión predijeron este criterio. Este resultado se podría explicar teniendo en cuenta el alto porcentaje de personas casadas que había en la muestra (77,6 \%), estado éste que podría estar actuando como un factor de protección, ante los cambios negativos que se producen en el apoyo social cuando aparece la ECV (Grace et al., 2002).

La presente investigación cuenta con una serie de limitaciones que es preciso comentar. En primer lugar, dado que el diseño de esta investigación fue de carácter transversal, sería conveniente contrastar los resultados obtenidos con otros procedentes de estudios longitudinales. De esta forma se podrían valorar los posibles cambios en las variables estudiadas, así como su efecto sobre el ajuste a la enfermedad a lo largo del tiempo.

En segundo lugar, la inclusión de otros elementos, como el estudio de las consecuencias que puede tener la ocurrencia de un único problema cardiovascular, frente a un mayor grado de comorbilidad, o la influencia de la historia y la evolución de la enfermedad de cada paciente, así como el tipo y el número de medicamentos prescritos, representan aspectos de interés para comprender las posibles diferencias en la adaptación a la ECV.

Por otro lado, el presente trabajo confirma que la ira y depresión pueden tener un papel fundamental en la aparición de factores de riesgo ya preestablecidos, como los de tipo comportamental o biológico. Sin embargo, algunos de nuestros resultados son discrepantes con los obtenidos en este sentido, por otras investigaciones. Así, el mayor nivel de cumplimiento de la dieta o el hecho de dejar de fumar cuando el nivel de depresión aumenta, condu- 
ce a plantear investigaciones adicionales con las que se intente esclarecer los mapas de interrelación entre las variables estudiadas, así como su influencia directa o indirecta sobre los diferentes hábitos comportamentales.

Finalmente, desde el punto de vista metodológico, habría que mencionar la baja fiabilidad obtenida con algunas de las escalas de afrontamiento, incluidas en la versión breve del COPE. Esta limitación podría explicarse si se tiene en cuenta que cada escala estaba compuesta de dos ítems. No obstante, hay que señalar que en otros estudios (Murberg y Bru, 2001) se han obtenido, con esta versión del COPE, coeficientes de fiabilidad adecuados.

En resumen, los resultados presentados en este estudio contribuyen a ampliar la investigación enmarcada en el contexto de la enfermedad cardiovascular.

Por una parte, apoyan la existencia de diferencias de sexo en relación con determinados aspectos vinculados con la ECV. En particular ponen de relieve que ciertos factores de riesgo comportamentales, como el consumo de tabaco y de alcohol, el estado de ánimo, la calidad de vida y el tipo de patología cardiovascular padecido, varían en pacientes con enfermedad cardiovascular en función de su sexo. La constatación de estas diferencias plantea, por lo tanto, la necesidad de orientar y diseñar un tipo de intervención especializada, en donde se aborden las necesidades y características particulares del paciente teniendo en cuenta su sexo.

Por otra parte, el estudio destaca la relevancia que tiene la depresión, frente a la ira, en el ajuste y pronóstico de la ECV. Si bien la ira se asoció con un mayor riesgo de consumir tabaco, la depresión predijo una mayor probabilidad de padecer algún tipo de trastorno cardiovascular. La depresión, además, se relacionó, en mayor medida que la ira, con un afrontamiento de la enfermedad orientado a una regulación inadecuada de las emociones y con un mayor decremento en la calidad de vida de los pacientes; siendo las áreas más afectadas las relativas al bienestar físico y psicológico, y la satisfacción personal.

\section{Bibliografía}

Abbey, S. E., \& Stewart, D. E. (2000). Gender and psychosomatic aspects of ischemic heart disease. Journal of Psychosomatic Research, 48, 417-423.

Angerer, P., Siebert, U., Kothny, W., Mühlbauer, D., Mudra, H., \& von Schacky C. (2000). Impact of social support, cynical hostility and anger expression on progression of coronary atherosclerosis. Journal of American College of Cardiology 36, 1781-1788.

Birket-Smith, M., Hansen, B. H., Hanash, J. A., Hansen, J. F., \& Rasmussen, A. (2009). Mental disorders and general well-being in cardiology outpatients-6 year survival. Journal of Psychosomatic Research, 67, 5-10.

Blom, M., Georgiades, A., Laszlo, K, Alinaghizadeh, H., Janszdy, I., \& Ahnve, S. (2007). Work and marital status in relation to depressive symptoms and social support among women with coronary artery disease. Journal of Women's Health, 16, 1305-1316.

Boutin-Foster, C., \& Charlson, M. E. (2007). Do recent life events and social support explain gender differences in depressive symptoms in patients who had percutaneus transluminal coronary angioplasty? Journal of Women's Health, 16, 114-123.

Boyle, S. H., Williams, R. B., Mark, D. B., Brummett, B. H., Siegler, I. C., Helms, M. J., \& Bare- 
foot, J. C. (2004). Hostility as a predictor of survival in patients with coronary artery disease. Psychosomatic Medicine 66, 629-632.

Boyle, S. H., Michalek, J. E., \& Suarez, E. C. (2006). Covariation of psychological attributes and incident coronary heart disease in U.S. air force veterans of Vietnam war. Psychosomatic Medicine 68, 844-850.

Boyle SH., Jackson WG. y Suarez EC. (2007). Hostility, anger, and depression predict increases in C3 over 10 years period. Brain, Behavior and Immunity, 21, 816-823.

Bromberger, J.T., \& Matthews, K. A. (1996). A feminine model of vulnerability to depressive symptoms: a longitudinal investigation of middle-aged women. Journal of Personality and Social Psychology, 70, 591-598.

Bunde, J., \& Suls, J. (2006). A quantitative analysis of the relationship between the CookMedley hostility scale and traditional coronary artery disease risk factors. Health Psychology, 25, 493-500.

Bute, B. P., Mathew, J., Blumenthal, J. A, WelshBohmer, K. \& investigadores de The Duke Heart Center. (2003). Female gender is associated with impaired quality of life 1 year after coronary artery bypass surgery female. Psychosomatic Medicine 65, 944-951.

Cahrart, R. L. y Ades, P. A. (1998). Gender differences in cardiac rehabilitation. Cardiology Clinics 16, 37-43.

Cameron, J. J. (2009). Depressive symptoms, depressive symptom types, and coping strategies among cardiac rehabilitation participants. Dissertations Abstracts International: Section B: The Sciences and Engineering, 69, 8-B, 5014.

Carver, C. S., Scheier, M. F., \& Weintraub, J. K. (1989). Assessing coping strategies: a theoretically based approach. Journal of Personality and Social Psychology, 56, 267-283.

Carver, C. S. (1997). You want to measure coping but your protocol's too long: Consider the Brief Cope. International Journal of Behavioral Medicine, 4, 92-100.
Chung, M. L., Moser, D. K., Lennie T., \& Rayens, M. (2009). The effects of depressive symptoms and anxiety on quality of life in patients with heart failure and their spouses: testing dyadic dynamics using actor-partner interdependence model. Journal of Psychosomatic Research, 67, 29-35.

Davidson, K. W., Kupfer, D. J., Bigger, J. T., Califf, R. M., \& investigadores de The National Heart Lung and Blood Institute Working Group Report. (2006). Assessment and treatment of depression in patients with CVD. Psychosomatic Medicine 68, 645-650.

Derogatis L. R. (1977). Administration, Scoring \& Procedures. Manual for the revised version of SCL-90. Baltimore. Johns Hopkins University Press.

Doering, L. V., Dracup, K., Caldwell, M. A., Moser, D. K., Erickson, V. S., Fonarow, G., \& Hamilton M. (2004). Is coping style linked to emotional states in heart failure patients?. Journal of Cardiac Failure, 10, 4, 344-349.

Donker, F. J., Breteler, M. H., \& van der Staak, C. P. (2000). Assessment of hostility in patients with coronary heart disease. Journal of Personality Assessment, 75, 1, 158-177.

Eaker, E. D., Sullivan, L. M., Kelly-Hayes, M., D'Agostino, R. B., \& Benjamin, E. J. (2004). Anger and hostility predict the development of atrial fibrillation in men in the Framingham Offspring Study. Circulation, 109, 1267-1271.

Emery, C. F, Frid, D. J., Engebretson, T. O., Alonzo, A. A., Fish, A., Ferketich, A. K., ... Stern, S. L. (2004). Gender differences in quality of life among cardiac patients. Psychosomatic Medicine 66, 190-197.

Everson, S. A., Goldberg, D. E., Kaplan, G. A., Julkunen, J.; \& Salonen, J. T. (1998). Anger expression and incident hypertension. Psychosomatic Medicine 60, 730-735.

Everson, S. A., Kaplan, G. A., Goldberg, D. E., Lakka, T. A., Sivenius, J., \& Salonen, J. T. (1999). Anger expression and incident stroke: prospective evidence from the Kuo- 
pio isquemic heart disease study. Stroke 30, 523-528.

Everson-Rose, S. A., \& Lewis, T. T. (2005). Psychosocial factors and cardiovascular diseases. Annual Review of Public Health 26, 469-500.

Faller, H., Störk, S., Schowalter, M., Steinbüchel, T., Wollner, V., Ertl, G., \& Angermann CE. (2007). Is health-related quality of life an independent predictor of survival in patients with chronic heart failure? Journal of Psychosomatic Research, 63, 533-538.

Fenton, W. S., \& Stover, E. S. (2006). Mood disorders: Cardiovascular and diabetes comorbidity. Current Opinion in Psychiatry, 19, 421-427.

Fernández-Abascal, E. G., Martín, M. D. \& Domínguez FJ. (2003). Factores de riesgo e intervenciones psicológicas eficaces en trastornos cardiovasculares. Psicothema, 15, 615-630.

Fillion, L., Kovacs, A. H., Gagnon, P., \& Endler, N. S. (2002). Validation of the shortened COPE for use with breast cancer patients undergoing radiation therapy. Current Psychology 21, 17-34.

Fontana, A., Pontari, B., \& Nash, D. (1998). Coping and parental history of cardiovascular disorders. Influence blood pressure in women tested during different phases of their menstrual cycles. Behavioral Medicine 23, 179-188.

Frasure-Smith, N., Lespérance, F., \& Talajic, M. (1995). The impact of negative emotions on prognosis following myocardial infarction: Is it more than depression? Health Psychology, 14, 388-398.

Frasure-Smith N., \& Lespérance F. (2005). Reflections on depression as a cardiac risk factor. Psychosomatic Medicine, 68, 651-656.

Frasure-Smith, N., \& Lespérance, F. (2008). Depression and anxiety as predictors of 2 year cardiac events in patients with stable coronary artery disease. Archives of General Psychiatry, 65, 62-71.
Fredrikson, B. L., Maynard, K. E., Helms, M. J., Haney T. L., Siegler, I. C., \& Barefoot, J. C. (2000). Hostility predicts magnitude an duration of blood pressure responses to anger. Journal of Behavioral Medicine, 23, 229-243.

Giltay, E. J., Kamphuis, M. H., Kalmijn, S., Zitman, F., \& Kromhout D.(2006). Disposicional optimism and risk of cardiovascular death. Archives of Internal Medicine 166, 431-436.

González de Rivera, J. L., Derogatis, L. R., GarcíaMarco, R., Rodríguez, F., Henry, M., \& Monterrey, A. (1989). The Spanish version of the SCL-90-R. Normative data in the general population. Clinical Psychometric Research, Towson MD.

Goodwin, R. D., \& Gotlib, H. (2004). Gender differences in depression: The role of personality factors. Psychiatry Research, 126, 135142.

Grace, S. L., Krepostman S., Brooks, D., Arthur, H., Scholey, P., Suskin, P., Jaglal, S., Abramson, B. L., \& Stewart, D. E. (2002). Illness perceptions among cardiac patients: Relation to depressive symptomatology and sex. Journal of Psychosomatic Research, 59, 153160.

Gremigni, P., Bettinardi, O., \& Bitti, P. E. (2000). Dimensioni personali e stili di coping in pazienti cardiooperati. Psicoterapia Cognitiva e Comportamentale, 6, 219-231.

Helmers, K. F., \& Krantz, D. S. (1996). Defensive hostility, gender and cardiovascular levels and responses to stress. Annals of Behavioral Medicine 18, 246-254.

Holahan, C. J., Moos, R. H., Holahan, C. K., \& Brennan, P. L. (1997). Social context, coping strategies and depressive symptoms. An expanded model with cardiac patients. Journal of Personality Social Psychology, 72, 918-928.

Ibishi, N. F., Musliu, N. R., Kamberi, A., Qirko, S., Brokaj, S., Lezha, M.,... Masala J. (2009). Impact of depression and cardiovascular disease. Psychiatric Annals, 39, 22-25. 
Jacka, F. N., Pasco, J. A., McConnell, S., Williams, L. J., Kotowicz, MA., Nicholson, G. C., \& Berk, M. (2007). Self-reported depression and cardiovascular risk factors in a community sample of women. Psychosomatics 48, 54-59.

Juárez García, A. (2008). La perspectiva multinivel en las enfermedades cardiovasculares: el rol de las características psicosociales del trabajo. Psicologia.com, 12(1). Recuperado de http://www.psiquiatria.com/revistas/ index.php/psicologiacom/article/viewArticle/726/.

Julkunen, J., \& Ahlström, R. (2006). Hostility, anger, and sense of coherence as predictors of health-related quality of life. Results of an ASCOT substudy. Journal of Psychosomatic Research 61, 33-39.

Kao, C. W. (2007). Quality of life and physiological status as predictors of mortality in patients with ICD. Dissertation Abstracts International: Section B: Sciences \& Engineering $67,4107$.

Kristofferzon, ML., Löfmark, R., \& Carlsson M. (2003). Myocardial Infarction: Gender differences in coping and social support. Journal of Advanced Nursing, 44, 360-374.

Kristofferzon, M. L., Löfmark, R., \& Carlsson, M. (2005). Coping, social support and quality of life over time after myocardial infarction. Journal of Advanced Nursing 52, 113-124.

Kubzansky, L. D., \& Kawachi, I. (2000). Going to the heart of the matter: do negative emotions cause coronary heart disease? Journal of Psychosomatic Research 48, 323-337.

Lampert, R., Joska, T., Burg, M., Batsford, W. P., McPherson, C. A., \& Jain D. (2002). Emotional and physical precipitants of ventricular arrhythmia. Circulation, 106, 1800-1805.

Lane, D., Carroll, D., Ring, C., Beevers, D. G., \& Lip, G. Y. (2001). Mortality and quality of life 12 months after myocardial infarction: Effects of depression and anxiety Psychosomatic Medicine, 63, 221-230.
Lavie, C. J., \& Milani, R. V. (2005). Prevalence of hostility in young coronary artery disease patients and effects of cardiac rehabilitation and exercise training. Mayo Clinic Proceedings 70, 335-342.

Leong J., Molassiotis A., \& Marsh, H. (2004). Adherence to health recommendations after cardiac rehabilitation programme in postinfarction patients: the role of health belie$\mathrm{fs}$, locus of control and psychological status. Clinical Effectiveness in Nursing, 8, 26-38.

Lett, H. S., Blumenthal, J. A., Babyak, M. A., Sherwood, A., Strauman, T., Robins, C., \& Newman, M. F. (2004). Depression as a risk factor for coronary artery disease: evidence, mechanisms and treatment. Psychosomatic Medicine 66, 305-315.

Lin, M. F., Ko, H. C., Wu, J. Y., \& Chang, F. M. (2008). The impact of extroversion or menopause status on depressive symptoms among climacteric women in Taiwan: Neuroticism as moderator or mediator. Menopause, 15, 138-143.

Lowe, R., Norman, P., \& Bennett, P. (2000). Coping emotion and perceived health following myocardial infarction : Concurrent and predictive associations. British Journal of Health Psychology, 5, 337-350.

Matthews, K. A., Gump, B. B., Harris, K. F., Haney, T. L., \& Barefoot JC. (2004). Hostile behavior predict cardiovascular mortality among men enrolled in the multiple risk factor intervention trial. Circulation 109, 66-70.

Mayou, R. A. (2000). Depression and anxiety as predictors of outcome after myocardial infarction. Psychosomatic Medicine, 62, 212219.

Murberg, T. A., \& Bru, E. (2001). Coping and mortality among patients with congestive heart failure. International Journal of Behavioral Medicine, 8, 66-79.

Murberg, T. A., Furze, G., \& Bru, E. (2004). Avoidance coping styles predict mortality among patients with congestive heart failure: A 6-year follow-up study. Personality and Individual Differences, 36, 757-766. 
Organización Mundial de la Salud: Enfermedades cardiovasculares. Recuperado de WWW.who.int/topics/cardiovascular_diseases/es/.

Penninx, B. W., Beekman, A. T., Honig, A., Deeg, D. J., Schoevers, R. A., von Eijk, J. T., \& van Tilburg, W. (2001). Depression and cardiac mortality: Results from a community-based longitudinal study. Archives of General Psychiatry 58, 221-227.

Rose, M., Köhler, K., Köhler F., Sawitzky B., Fliege H., \& Klapp BF. (2005). Determinants of quality of life of patients with congenital heart disease. Quality of life Research, 14, 35-43.

Rueda, B.; \& Pérez-García, A. (2006). Gender and social support in the context of cardiovascular disease. Woman \& Health 43, 59-73.

Rueda, B., \& Pérez-García, A. (2006). A prospective study of the effects of psychological resources and depresión in esencial hypertension. Journal of Health Psychology, 11, 129-140.

Ruiz, M. A., \& Baca, E. (1993). Design and validation of the quality of life questionnaire. A generic health-related quality of life instrument. European Journal of Psychological Assessment, 9, 19-32.

Ruiz, M. I., Lopez, N., Antuña, P. \& García, J. (2008). Atención clínica a pacientes con factores emocionales asociados a enfermedad coronaria, desde un programa de psiquiatría de enlace. Interpsiquis, 1. Recuperado de http://www.psiquiatria.com/articulos/ psiq_general_y_otras_areas/psiq_de_enlace/34411/.

Sasaki, M.; \& Yamasaki K. (2002). Causal relationship between hostility and health status and the mediating role of stress coping. Japanese Journal of Health Psychology, 15, 1-11.

Siegler, I. C., Peterson, B. L, Barefoot, J. C., \& Williams, R. B. (1992). Hostility during late adolescence predicts coronary risk factors al midlife. American Journal of Epidemiology, 136, 146-154.
Shen, B. J. (2002). Psychosocial predictors in the recovery and rehabilitation of patients with coronary heart disease. Dissertation Abstracts International: Section B: Sciences \& Engineering 62, 11-B, 5392.

Shen, B. J., McCreary, C. P., \& Myers, H. F. (2004). Independent and mediated contributions of personality, coping, social support and depressive symptoms to physical functioning outcome among patients in cardiac rehabilitation. Journal of Behavioural Medicine 27, 39-62.

Smith, T. W., Glazer, K., Ruiz, J. M., \& Gallo, L. C. (2004). Hostility, anger, aggressiveness, and coronary heart disease: an interpersonal perspective on personality, emotion and health. Journal of Personality, 72, 12171270.

Stafford, L., Berk, M., Reddy, P., \& Jackson, H. J. (2007). Comorbid depression and healthrelated quality of life in patients with coronary artery disease. Journal of Psychosomatic Research, 62, 401-410.

Strik, J. J., Honig, A., Lousberg, R., \& Denollet, J. (2001). Sensibility and specificity of observer and self-report questionnaire in major and minor depression following myocardial infarction. Psychosomatics 42, 423-428.

Vandervoort, D. J. (1992). Belief systems and coping styles as mediating variables in the relationship between hostility and illness. Current Psychology, 11, 226-235.

Vandervoort, D. J. (2006). Hostility and health: Mediating effects of belief systems and coping styles. Current Psychology, 25, 50-66.

Weidner, G. (2000). Why do men get more heart disease than women?. An international perspective. Journal of American College Health 48, 291-294.

Whitmarch, A., Koutantji, M., \& Sidell, K. (2003). Illness perceptions mood and coping at cardiac rehabilitation. British Journal of Health Psychology, 8, 209-221. 
Wielgosz, A. T., \& Nolan, R. P. (2000). Biobehavioral factors in the context of ischemic cardiovascular diseases. Journal of Psychosomatic Research 48, 339-345.

Wulsin, L. R., \& Singal, B. M. (2003). Do depressive symptoms increase the risk for the onset of coronary disease? A systematic quantitative review. Psychosomatic Medicine 65, 201-210.

Zeidner, M., \& Saklofske, D. (1996). Adaptive and maladaptive coping. En M. Zeidner, \& N.S. Endler (Eds.), Handbook of coping: Theory, research and applications (pp.3-23). New York: John Wiley and Sons. 